\title{
Early detection of human glioma sphere xenografts in mouse brain using diffusion MRI at 14.1T
}

\author{
P. Porcari ${ }^{1,2} \mid$ M. E. Hegi ${ }^{3} \mid$ H. Lei ${ }^{1,4} \mid$ M-F. Hamou ${ }^{3} \mid$ I. Vassallo ${ }^{3} \mid$ S. Capuani ${ }^{5}$ | \\ R. Gruetter ${ }^{1,4,6,7}$ | V. Mlynarik ${ }^{1,8}$
}

${ }^{1}$ Centre for Biomedical Imaging, Ecole Polytechnique Fédérale de Lausanne, Lausanne, Switzerland

${ }^{2}$ Newcastle Magnetic Resonance Centre, Newcastle University, Newcastle Upon Tyne UK

${ }^{3}$ Laboratory of Brain Tumor Biology and Genetics, Service of Neurosurgery and Neuroscience Research Centre, Department of Clinical Neurosciences, Lausanne University Hospital and University of Lausanne, Lausanne, Switzerland

${ }^{4}$ Department of Radiology, University of Geneva (UNIGE), Geneva, Switzerland

${ }^{5}$ CNR-ISC UOS Roma Sapienza, Physics Department, Sapienza University of Rome, Rome, Italy

${ }^{6}$ Department of Radiology, University of Lausanne, Lausanne, Switzerland

${ }^{7}$ LIFMET, Ecole Polytechnique Fédérale de Lausanne, Lausanne, Switzerland

${ }^{8}$ High Field MR Center, Medical University of Vienna, Vienna, Austria

\section{Correspondence}

P. Porcari, Newcastle Magnetic Resonance Centre, Newcastle University, Newcastle Upon Tyne, UK.

Email: paola.porcari@ncl.ac.uk
Glioma models have provided important insights into human brain cancers. Among the investigative tools, MRI has allowed their characterization and diagnosis.

In this study, we investigated whether diffusion MRI might be a useful technique for early detection and characterization of slow-growing and diffuse infiltrative gliomas, such as the proposed new models, LN-2669GS and LN-2540GS glioma sphere xenografts. Tumours grown in these models are not visible in conventional $T_{2}$-weighted or contrast-enhanced $T_{1}$-weighted $M R I$ at 14.1 T.

Diffusion-weighted imaging and diffusion tensor imaging protocols were optimized for contrast by exploring long diffusion times sensitive for probing the microstructural alterations induced in the normal brain by the slow infiltration of glioma sphere cells.

Compared with $T_{2}$-weighted images, tumours were properly identified in their early stage of growth using diffusion MRI, and confirmed by localized proton MR spectroscopy as well as immunohistochemistry. The first evidence of tumour presence was revealed for both glioma sphere xenograft models three months after tumour implantation, while no necrosis, oedema or haemorrhage were detected either by MRI or by histology. Moreover, different values of diffusion indices, such as mean diffusivity and fractional anisotropy, were obtained in tumours grown from LN-2669GS and LN-2540GS glioma sphere lines. These observations highlighted diverse tumour microstructures for both xenograft models, which were reflected in histology. This study demonstrates the ability of diffusion MRI techniques to identify and investigate early stages of slow-growing, invasive tumours in the mouse brain, thus providing a potential imaging biomarker for early detection of tumours in humans.

\section{KEYWORDS}

ADC, brain tumours, diffusion MRI, FA, human glioma xenografts, immunohistochemistry, MD, MRS

\section{1 | INTRODUCTION}

Diffuse gliomas (World Health Organization (WHO) Grade II-IV) ${ }^{1}$ are the most common primary brain tumours in humans and one of the leading causes of cancer-related deaths in adults. ${ }^{2}$ Their diffuse infiltration into the surrounding normal brain precludes complete resection and they all eventually recur, usually having progressed to a more aggressive tumour. The infiltrative part will give rise to tumour recurrence even in $\mathrm{MRI}$-controlled, macroscopically fully resected patients, since the extent of invasion is not visible using conventional $T_{1}$ and $\mathrm{T}_{2}$-weighted MRI. Hence, it is difficult to target treatment to this 'invisible' part. Among diffuse gliomas, glioblastoma (GBM; WHO Grade IV) is the most aggressive form, ${ }^{3}$ with a median overall survival of only 14.6 months ${ }^{4}$ despite multimodal treatment of maximal safe resection, followed by combined chemo-radiotherapy. Visualization of the infiltrative portion of gliomas is a necessary first step for testing novel treatment strategies affecting migrating tumour cells. ${ }^{5}$

Over the past decades, rodent models of diffuse infiltrative gliomas have been extensively investigated ${ }^{6-8}$ to better understand the genetic and molecular pathology of malignant gliomas and to assess the efficacy of newer therapies. ${ }^{9}$ Although none of the existing models fully reflect the corresponding human neoplasm, ${ }^{7}$ their use in 
combination with advanced neuroimaging techniques ${ }^{10-12}$ has led to the identification of imaging biomarkers for the detection of brain cancers and early prediction of therapeutic efficacy. ${ }^{13}$

$\mathrm{MRI}$ allows non-invasive in vivo monitoring of both tumour growth and response to therapy, and provides detailed structural information on tumour and brain tissue. ${ }^{14}$ Morphological features of diffuse gliomas, e.g. tumour location and size, necrosis, peritumoral oedema, heterogeneity, and haemorrhagic foci, can be determined using routine contrast-enhanced $T_{1^{-}}$and $T_{2}$-weighted MRI. Nevertheless, conventional MRI has limited sensitivity and specificity in evaluating early therapeutic effects, in determining histological type and tumour grading and in accurately delineating tumour extent. ${ }^{15}$ Contrast-enhanced $T_{1}$-weighted MRI tends to underestimate the diffuse infiltration of glioma cells ${ }^{16}$ depending on the blood-brain-barrier integrity at the infiltrative margins. ${ }^{17}$ In addition, histological correlation with $T_{2}$-weighted images of gliomas has shown glioma-infiltrating cells beyond the hyper-intense region on $T_{2}$-weighted images. ${ }^{18}$

To overcome these limitations, advanced MRI methods sensitive to tissue properties related to brain cancer biology and glioma cell migration have been developed and implemented. ${ }^{14,19}$ Among these, diffusion $\mathrm{MRI},{ }^{20}$ which reflects information on the tissue microstructure, potentially allows imaging of glioma-infiltrating cells in the normal brain $^{21}$ and the detection of early changes in the tumour microenvironment following treatment. ${ }^{22}$ The diffusion-weighted MR signal, which is recorded as a function of diffusion weighting or $b$ value, is affected by the complex composition and the geometric architecture of the investigated tissues. Due to the presence of structural barriers, water diffusion in brain tissue is restricted and/or hindered. Therefore, the diffusion time $(\Delta)$ becomes a key parameter for improving the sensitivity of diffusion MRI to tissue microstructure. For longer $\Delta$, water molecules in the brain probe more obstacles and/or barriers over larger spatial scales, which are, however, smaller than the dimensions of a pixel. Moreover, with increasing $\Delta$, diffusion can be considered 'quasi-Gaussian' in normal physiological conditions, and the measured diffusion coefficient shows a rather monotonic behaviour as function of $\Delta$ and membrane permeability, ${ }^{23}$ which is more visible for shorter diffusion times. ${ }^{24}$ Therefore, the measured diffusion coefficient, which reflects tissue characteristics, might change with the tumour progression for longer $\Delta$, thus playing an important role in evaluating healthy and diseased tissues. To our knowledge, no previous studies have reported the effect of $\Delta$ on water diffusion in human glioma models, except an in vitro study on glioma cells. ${ }^{25}$ By increasing the diffusion time, water molecules probe a wider area of tissue in accordance with the relation $\left(I=(2 D \Delta)^{1 / 2}\right)$ between the diffusion distance $(I)$ and the diffusion time $(\Delta)$. As a consequence, we hypothesize that the diffusion signal at extended $\Delta$ will be more sensitive to the small changes in cell density that are characteristic of the slow infiltration of gliomas. This feature is well modelled in the investigated xenografts.

Currently, diffusion-weighted imaging (DWI) ${ }^{26}$ and diffusion tensor imaging (DTI) ${ }^{27,28}$ techniques are commonly used to investigate diffuse infiltrative gliomas in both preclinical ${ }^{29,30}$ and clinical settings. ${ }^{31,32}$ These techniques allow the detection of the invasive pathways of glioma cell migration in the brain, which seem to preferentially occur along the myelinated fibres in white matter tracts. $^{1,33}$
Using optimized diffusion protocols at $14.1 \mathrm{~T}$, the aim of this study was to identify slow-growing glioma sphere xenografts in their early stages of growth, which are otherwise not visible using conventional Gd-DTPA enhanced $T_{1}$-weighted ${ }^{34}$ and $T_{2}$-weighted MRI. In addition, differences in diffusion indices of LN-2669GS and LN-2540GS glioma sphere xenografts allowed the evaluation of differences in their microstructure. Tumour growth detected by diffusion methods was confirmed by localized proton MRS and immunohistochemistry.

\section{2 | MATERIALS AND METHODS}

\section{1 | Glioma-derived sphere lines}

Two human glioma-derived sphere lines, LN-2540GS and LN$2669 \mathrm{GS},{ }^{35,36}$ were used for orthotopic xenografts in nude mice. As previously described, ${ }^{36}$ each line was derived by mechanical and enzymatic dissociations of GBM surgical specimens into a single cell suspension. After a CD133 (Miltenyi Biotec, Bergisch Gladbach, Germany) selection procedure, cells were cultured under stem cell conditions in DMEM-F12 medium (Invitrogen, Carlsbad, CA, USA; 10565-018) supplemented with 2\% of B27 (Invitrogen; 17504), human recombinant epidermal growth factor $(20 \mathrm{ng} / \mathrm{ml})$ and human recombinant basic fibroblast growth factor $(20 \mathrm{ng} / \mathrm{ml})$ (PeproTech, Rocky Hill, NJ, USA; AF-100-15 and 100-18B).

Basic genetic characterization and authentication have been published previously. ${ }^{35,36}$

\section{2 | Animal model}

Orthotopic human gliomas were induced in eight female athymic nude mice (Swiss nu/nu; Charles River Laboratories France, L'Arbresle, France), aged 6-9 weeks and weighing 20-28 g. All experimental procedures and animal care were performed according to the federal and local ethical guidelines and approved by the local veterinary authorities (VD 1181-5). Mice were anesthetized and placed into a stereotaxic frame (Stoelting, Wood Dale, IL, USA). Glioma sphere cells $\left(1 \times 10^{5}\right)$, dissociated into a single cell suspension, were injected in a volume of $5 \mu \mathrm{l}$ of PBS via a stereotaxic injector at a flow rate of $5 \mu \mathrm{l} / \mathrm{min}$ (Quintessential Stereotaxic Injector $(\mathrm{QSI})^{\mathrm{TM}}$, Stoelting) into the right striatum of the mice (coordinates from bregma: $0.0 \mathrm{~mm}$ posterior, $2.5 \mathrm{~mm}$ lateral, $3.0 \mathrm{~mm}$ ventral; $n=4$ per sphere line).

\section{3 | Animal preparation for MRI}

Mice underwent MRI for the first time 50 days after orthotopic implantation of human glioma sphere cells and then once a week. Control mice $(n=4)$ were also investigated using the same MRI protocol.

For MRI measurements, each mouse was anaesthetized with isoflurane (4\% induction, $1.5-2 \%$ maintenance) in a mixture of air: $\mathrm{O}_{2}$ (1:0.5 $\mathrm{l} / \mathrm{min}$ induction, 0.7:0.3 $\mathrm{l} / \mathrm{min}$ maintenance), and placed in a home-built dedicated holder with the head stereotaxically fixed by ear pins and bite bar. Physiological monitoring was performed using an MRI-compatible small animal monitoring and gating system (Model 1025, SA Instruments, Stony Brook, NY, USA). The body temperature was maintained in the range $36.5-37.5^{\circ} \mathrm{C}$ using a circulating warm 
water system, and the respiratory rate kept between 60 and 90 bpm by regulating the isoflurane level.

\section{4 | MRI}

All measurements were performed on a $14.1 \mathrm{~T} / 26 \mathrm{~cm}$ horizontal bore magnet (Magnex Scientific, Oxford, UK) interfaced to a Direct Drive console (Varian Medical System, Palo Alto, CA, USA), and equipped with a $12 \mathrm{~cm}$ inner diameter actively shielded gradient set (400 mT/ $\mathrm{m}$ in $120 \mu \mathrm{s}$ ). A two-loop quadrature surface coil was used as an RF transceiver for MRI and MRS. MRI protocol included $T_{2}$-weighted images, DWI and DTI scans. After measuring scout scans, multi-slice $T_{2}$-weighted fast spin-echo images of mouse brain were acquired in the coronal orientation as an anatomical reference $\left(T_{\mathrm{R}} / T_{\text {eff }}=5000 /\right.$ $52 \mathrm{~ms}$, echo train length $=8$, field of view $=20 \times 20 \mathrm{~mm}^{2}$, matrix size $=256 \times 256$, in-plane resolution $=78 \times 78 \mu \mathrm{m}^{2}, 13$ slices of $0.6 \mathrm{~mm}$ thickness, four averages, scan time $=10 \mathrm{~min}$ ). Static field homogeneity was optimized by adjusting all first and second order shims using an EPI version of FASTMAP (fast automatic shimming techniques by mapping along projections), ${ }^{37}$ resulting in water linewidths of $18-24 \mathrm{~Hz}$ over the selected volume of interest (VOI; $6 \times 5 \times 4.5 \mathrm{~mm}^{3}$ ) centred in the mouse brain.

Diffusion-weighted images of mouse brain were acquired in the coronal orientation using a pulse gradient stimulated echo (PGSTE) sequence $^{38}\left(T_{R} / T_{E}=4000 / 22 \mathrm{~ms}\right.$; field of view $=20 \times 10 \mathrm{~mm}^{2}$, matrix size $=128 \times 64$, in-plane resolution $=156 \times 156 \mu \mathrm{m}^{2}$, eight slices of $1 \mathrm{~mm}$ thickness sufficient to cover the whole tumour and the peritumoral areas, two averages, scan time $=67 \mathrm{~min}$ ).

To optimize DWI and DTI protocol for glioma detection, a pilot study was carried out on mice with LN-2540GS xenograft at early stages of tumour development by exploring several diffusion times ( $\Delta=40,60,80,100,150,220 \mathrm{~ms}$ ) with gradient duration $(\delta)$ equal to $4 \mathrm{~ms}$. For each $\Delta$, DWI data were acquired using eight $b$ values (294, $389,542,754,1024,1352,2185,2688 \mathrm{~s} / \mathrm{mm}^{2}$ ) with the diffusion gradient applied along the readout direction ( $X$ axis). The corresponding gradient amplitudes are summarized in Table 1 . It is of note that the calculation of $b$ values includes the contribution of imaging and crusher gradients, which lead to $b_{0}$ values for the unweighted diffusion images, as reported in Table 1. The contrast-to-noise ratio (CNR) of the tumour was calculated for each diffusion-weighted image (acquired at each specified $\Delta$ and $b$ value) as the difference between the mean signal from regions of interest (ROIs) drawn (as circular areas of the same size) in the contralateral area, $\langle C L\rangle$, and in the tumour, $\langle T\rangle$, divided by the standard deviation of the signal in the $\mathrm{CL}$ region, $\sigma(\mathrm{CL})$, $[C N R=(\langle C L\rangle-\langle T\rangle) / \sigma(C L)]$ (Table 2). The signal-to-noise ratio (SNR) of tumour was determined for each $\Delta$ on the $b_{0}$ image as the mean signal intensity of a ROI drawn in the tumour location $(\langle T\rangle)$ divided by the standard deviation $(\sigma)$ of a large ROI in the background noise $(N)$, that was visually devoid of artefacts (SNR $=\langle\mathrm{T}\rangle / \sigma(N))$.

Following the observation in this pilot study, the main DWI protocol for both glioma sphere-derived xenografts, LN-2669GS and LN$2540 G S$, used $\Delta=80 \mathrm{~ms}$ with all the above-specified $b$ values, which was justified by the pilot study CNR data (Table 2, Figure 1). The same DWI protocol was also carried out with diffusion gradients applied along the phase encoding ( $Y$-axis) and the slice selection ( $Z$-axis) directions.

CNR of tumour growing from both glioma sphere xenografts was also determined for $\Delta=80 \mathrm{~ms}$ as a function of $b$ values for the DWI data acquired during the last MRI session.

DTI data were collected by acquiring seven images, one $b_{0}$ image (no diffusion weighting, $b=0 \mathrm{~s} / \mathrm{mm}^{2}$ ) and six diffusion-weighted images, each of them with the same $b$ value of $1352 \mathrm{~s} / \mathrm{mm}^{2}$ (which was found to provide the best tumour contrast by exploring different $\Delta$ and $b$ values in DWI scans as shown in Table 2 ) and encoding gradients applied in six unique orientations ([1, 0, 0], [0, 1, 0], [0, 0, 1], [1/ $\sqrt{ } 2,1 / \sqrt{ } 2,0],[1 / \sqrt{ } 2,0,1 / \sqrt{ } 2],[0,1 / \sqrt{ } 2,1 / \sqrt{ } 2])$. The acquisition time was $58 \mathrm{~min}$.

Apparent diffusion coefficient (ADC) maps of mouse brain were computed by fitting, voxel by voxel, the signal intensity of the diffusion-weighted images as a function of $b$ values $(S(b)=S(0) \exp (-b$ $A D C)$ ).

Diffusion tensor maps, mean diffusivity (MD) and fractional anisotropy (FA), which reflect respectively the average diffusivity and the degree of anisotropy in each voxel, ${ }^{27}$ were computed by reconstructing the tensor. All diffusion maps, ADC, MD and FA, were computed using the FSL DTIFIT routine (FSL, Version 4 software; http://www.fmrib.ox.ac.uk/fsl).

ROls containing the tumour, a CL area and the corresponding brain regions of controls were drawn (as circular areas of the same size) on the ADC, MD and FA maps with reference to the diffusion-weighted

TABLE 1 DWI protocol used to optimize tumour visualization. Summary of diffusion gradient amplitudes $(G)$ corresponding to each $b$ value and diffusion time $(\Delta)$ used in the DWI acquisition

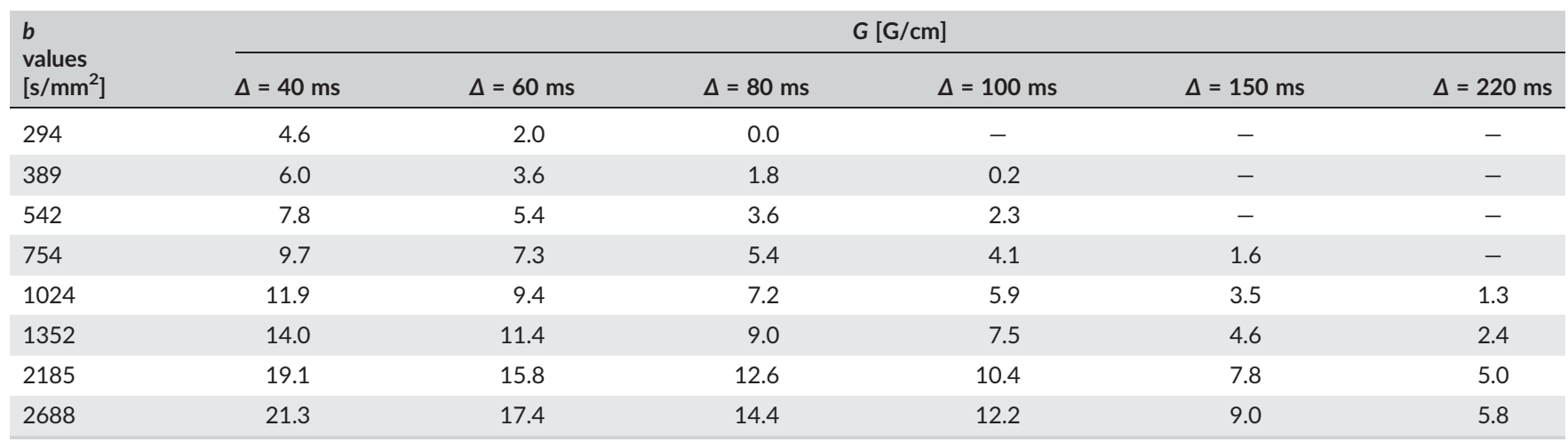

A missing value (-) means a $b_{0}$ value higher than the considered $b$ values $\left(b_{0}=381,590\right.$ and $884 \mathrm{~s} / \mathrm{mm}^{2}$ for $\Delta=100,150$ and $220 \mathrm{~ms}$, respectively). 
TABLE 2 Summary of CNR determined from DW images acquired with different $\Delta$ and $b$-values from mouse brain with slow growing and highly infiltrative tumour at early stage of growth

\begin{tabular}{|c|c|c|c|c|c|c|}
\hline \multirow{2}{*}{$\begin{array}{l}b \text { values } \\
{\left[\mathrm{s} / \mathrm{mm}^{2}\right]}\end{array}$} & \multicolumn{6}{|c|}{ CNR } \\
\hline & $\Delta=40 \mathrm{~ms}$ & $\Delta=60 \mathrm{~ms}$ & $\Delta=80 \mathrm{~ms}$ & $\Delta=100 \mathrm{~ms}$ & $\Delta=150 \mathrm{~ms}$ & $\Delta=220 \mathrm{~ms}$ \\
\hline 294 & $-0.43 \pm 0.08$ & $0.16 \pm 0.04$ & $0.54 \pm 0.10$ & - & - & - \\
\hline 542 & $0.52 \pm 0.04$ & $0.94 \pm 0.12$ & $1.61 \pm 0.08$ & $1.46 \pm 0.12$ & - & - \\
\hline 752 & $1.22 \pm 0.07$ & $1.44 \pm 0.09$ & $1.63 \pm 0.09$ & $1.66 \pm 0.06$ & $1.44 \pm 0.04$ & - \\
\hline 2185 & $0.98 \pm 0.09$ & $1.43 \pm 0.09$ & $1.98 \pm 0.10$ & $1.24 \pm 0.23$ & $1.01 \pm 0.09$ & $0.86 \pm 0.25$ \\
\hline 2688 & $0.71 \pm 0.10$ & $1.01 \pm 0.08$ & $1.44 \pm 0.09$ & $0.80 \pm 0.16$ & $0.37 \pm 0.09$ & $-0.16 \pm 0.03$ \\
\hline
\end{tabular}

All values are mean \pm SD.

A missing value (-) indicates no DW images were available for the evaluation of the CNR.
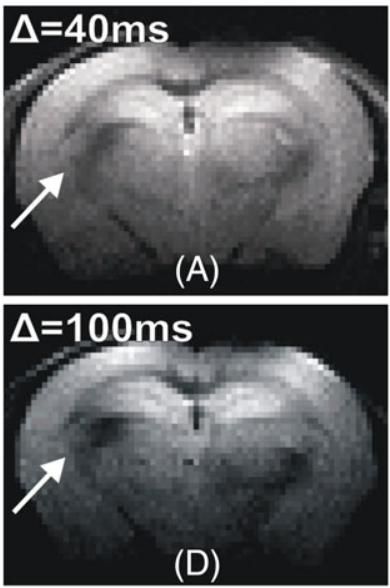
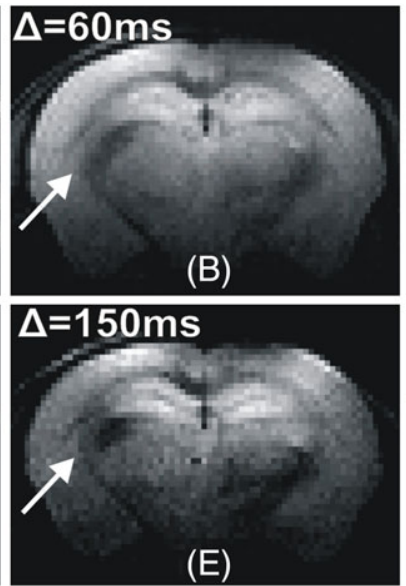

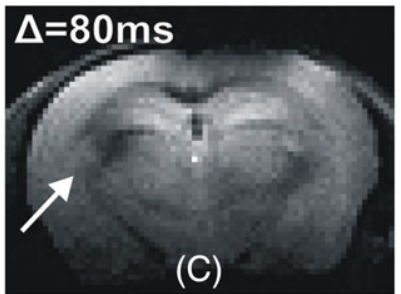

$\Delta=220 \mathrm{~ms}$

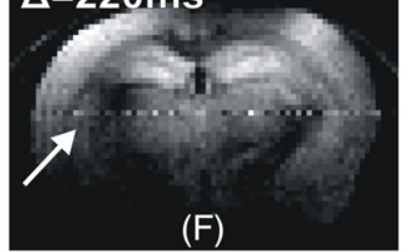

FIGURE 1 In vivo MRI of LN-2540GS glioma sphere xenograft, 80 days after cell injection (pilot study data). Diffusion-weighted images of a mouse brain with LN-2540GS glioma sphere xenograft acquired with different diffusion times $(\Delta=40,60,80,100,150,220 \mathrm{~ms})$ and the same $b$ value $\left(1352 \mathrm{~s} / \mathrm{mm}^{2}\right)$. C shows a slightly better tumour contrast compared with the others

image with $\Delta=80 \mathrm{~ms}, \mathrm{~b}=1352 \mathrm{~s} / \mathrm{mm}^{2}$, where tumour showed better contrast (Table 2). The $\mathrm{CL}$ area was selected considering the brain region symmetric to the tumour location with respect to the midsagittal plane. Mean values of ADC, MD and FA were calculated in the selected ROls using a custom MATLAB script.

Statistically significant difference between the mean values of diffusion indices calculated in the selected ROls of all tumour slices in all mice and in the corresponding CL was accepted for $P<0.05$, as determined with a two-tailed, paired Student's $t$ test. Comparison between the diffusion indices calculated in the tumour of both xenografts as well as the comparison between the diffusion indices calculated in the tumour of each xenograft and in the corresponding brain region of the control group were considered as statistically significant for $P<0.05$, as determined with a two-tailed, unpaired Student's $t$ test.

\section{5 | MRS}

Spectra of gliomas were acquired during the final MRI session in each mouse. Proton spectra were collected using a short echo-time $\left(T_{E}=2.8 \mathrm{~ms}\right)$ spin echo, full intensity acquired localized (SPECIAL) $)^{39}$ spectroscopy sequence from a VOI $\left(2 \times 2 \times 2 \mathrm{~mm}^{3}\right)$ located within the tumour, based on coronal diffusion-weighted images. Before measurements, field homogeneity was adjusted in the selected VOI, as mentioned above. Outer volume suppression interleaved with a VAPOR (variable power and optimized relaxation delays) ${ }^{40}$ water signal suppression was used. Spectra were acquired with $T_{R}=4000 \mathrm{~ms}$, 15 blocks of 16 transients (total scan time $16 \mathrm{~min}$ ), spectral width of $7 \mathrm{kHz}$ and 4096 data points. Each block was corrected for static field drift and summed for further analysis. Spectra from brain regions of healthy mice matching tumour size and location in the brain of injected mice were acquired for comparison.

Metabolite concentrations were quantified using LCModel $^{41}$ and absolute concentrations of metabolites were calculated using an unsuppressed water peak as a reference.

Statistically significant levels were accepted for $P<0.05$, as determined with a two-way ANOVA followed by the Bonferroni multi-comparison post-tests.

\section{6 | Immunohistochemistry}

All mice were sacrificed approximately 5 months after implantation, when gliomas were sufficiently large (maximum dimension of 2-3 $\mathrm{mm}$ ) in diffusion-weighted images. Brains were dissected, fixed in formalin (4\% buffered formalin) and embedded in paraffin. Tissue 
sections $(5 \mu \mathrm{m})$ were stained with H\&E (haematoxylin and eosin) or immunostained for the proteins GFAP (glial fibrillary acidic protein; G3893, Sigma-Aldrich, St. Louis, MO, USA), mindbomb E3 ubiquitin protein ligase 1 (MIB-1; monoclonal antibody (MAB), M7240, Dako, Glostrup, Denmark), p53 (MAB, Dako, M7001), ${ }^{36}$ using standard procedures (Ventana Medical System, Tucson, AZ, USA), and human nucleolin (hNCL, 1:200, $4^{\circ} \mathrm{C}$, overnight; ab13541, Abcam, Cambridge, $\mathrm{UK}$; does not react with mouse). For hNCL, the antigen retrieval procedure in citrate phosphate buffer $\left(\mathrm{pH}\right.$ 6.0) was performed at $95^{\circ} \mathrm{C}$ for $60 \mathrm{~min}$ followed by $8 \mathrm{~min}$ at $120^{\circ} \mathrm{C}$ (pressure cooker), and an additional blocking step of 15 min with Rodent Block M (catalogue no RBM 961, Biocare Medical, Concorde, CA, USA) was added to the standard procedure. Immunoreactivity was visualized using DAB (diaminobenzidine tetrahydrochloride).

\section{3 | RESULTS}

\section{1 | MRI results}

The diffusion time $\Delta=80 \mathrm{~ms}$ was found to be optimal for identifying the glioma sphere xenografts, as shown from the pilot study CNR data (Table 2, Figure 1). The extension of the lesions was not properly visualized using lower diffusion times, whereas higher $\Delta$ led to unacceptably low SNR.

Tumours grown as LN-2669GS and LN-2540GS glioma sphere xenografts in mouse brain were detected for the first time three months after glioma implantation, as shown in Figure 2 and Figure 3 , respectively. Each of the figures shows typical $T_{2}$-weighted and diffusion-weighted images (Figure $2 \mathrm{~A}, \mathrm{~B}$ and Figure $3 \mathrm{~A}, \mathrm{~B}$ ) acquired three months after cell injection and the calculated $A D C$, and DTI maps (Figure $2 \mathrm{C}-\mathrm{F}$, Figure $3 \mathrm{C}-\mathrm{F}$ ) of a coronal slice from the mouse brain with LN-2669GS NCH-1364 and LN-2540GS NCH-1365 glioma sphere xenograft, respectively.

In both figures, the hypo-intense region on the diffusion-weighted images (Figures 2B, 3B), which is just visible for the LN-2669GS $\mathrm{NCH}-1364$ xenograft (Figure 2B), and the corresponding hyper- intense area on the $A D C$ and $M D$ maps (Figure $2 C, D$, Figure $3 C, D$ ) as well as the hypo-intense region on the FA map (Figure 2E, Figure 3E), shows the extent of the tumour. In contrast, no lesion is visible in the $T_{2}$-weighted images (Figure 2A, Figure 3A).

Figure 4 and Figure 5 show coronal $T_{2}$-weighted and diffusionweighted images (Figure $4 A, B$ and Figure $5 A, B$ ) acquired five months after injection of the cells as well as the computed ADC and DTI maps (Figure $4 \mathrm{C}-\mathrm{F}$, Figure $5 \mathrm{C}-\mathrm{F}$ ) from the mouse brain of the same mice as displayed in Figure 2 and Figure 3, respectively. As with Figure 2 and Figure 3 , both xenografts are visible in the diffusion-weighted images (Figure 4B, Figure 5B) as well as in the ADC (Figure 4C, Figure $5 C$ ) and DTI maps (Figure 4D-F, Figure 5D-F), whereas no evidence of tumour is seen in the corresponding $T_{2}$-weighted image (Figure 4A, Figure 5A).

Compared with Figure 2 and Figure 3, tumours grown from both sphere lines are more visible in Figure 4 and Figure 5 at five months after cell injection, due to tumour development.

Figure 6 shows DWI acquisition on the mouse brain comprising the LN-2540GS xenograft NCH-1365 (A) as well as the CNR data for both xenografts (B). All data were acquired during the last MRI session.

The progression of diffusion-weighted images (Figure 6A, a-h), acquired two weeks after those presented in Figure 5, demonstrates an improved delineation of tumour margins when the $b$ value increases. In particular, the lesion becomes visible when $b$ is higher than $500 \mathrm{~s} / \mathrm{mm}^{2}$ (Figure 6A, c), and the higher the $b$ value the more noticeable the tumour (Figure 6A, c-h), which appears as a hypo-intense lesion on the diffusion-weighted images (Figure 6A, c-h) and as a hyperintense area in the corresponding ADC map (Figure 6A, k). Conversely, the lesion is not visible on the $T_{2}$-weighted image (Figure $6 \mathrm{~A}, \mathrm{j}$ ) that shows only a ventricular enlargement, as in Figure 5A.

The CNR data shown in Figure 6B are displayed as a function of $b$ values ( $\Delta=80 \mathrm{~ms}$ ) for images acquired in the last MRI session (Figure 4 and Figure $6 \mathrm{~A}$ ). The best tumour contrast is given for both xenografts at a $b$ value equal to $1352 \mathrm{~s} / \mathrm{mm}^{2}$, the same as given by the pilot study CNR data (Table 2).

Mean values of the diffusion indices calculated in the tumour $(T)$ and in the corresponding contralateral $(\mathrm{CL})$ area of each xenograft as
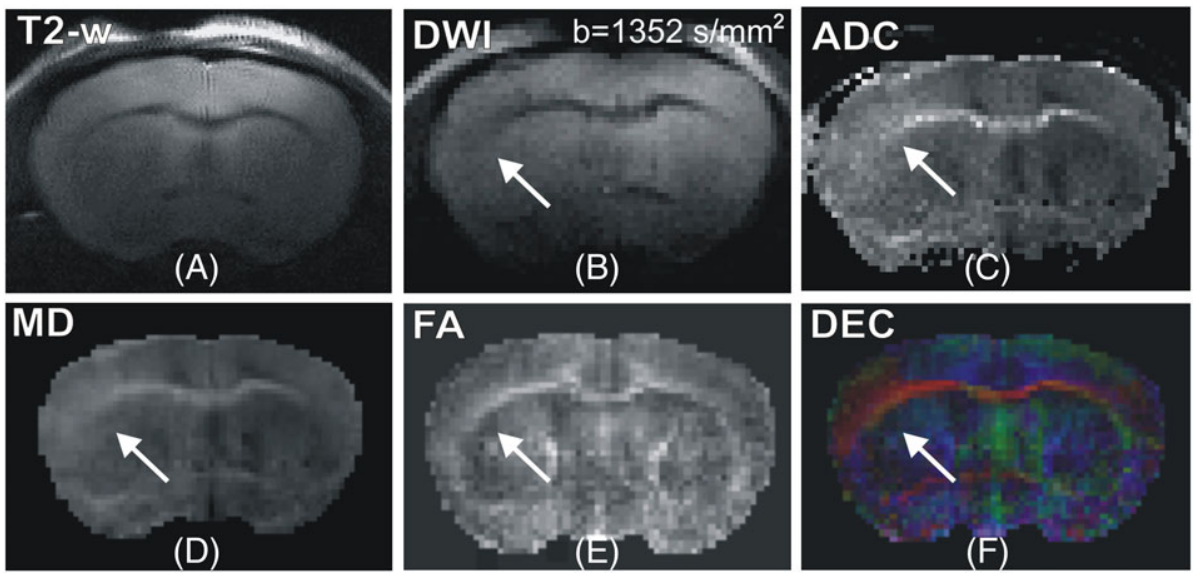

FIGURE 2 In vivo MRI of LN-2669GS glioma sphere xenograft, three months after cell injection. Upper panel: coronal $T_{2}$-weighted (A) and diffusion-weighted (B) images of the mouse brain with LN-2669GS xenograft NCH-1364, and the calculated ADC map (C). Lower panel: corresponding diffusion tensor maps, MD (D), FA (E), and FA-modulated directionally encoded colour (DEC) (F) maps. The lesion (arrow) is barely visible on the diffusion-weighted image (B), ADC (C) and DTI maps 

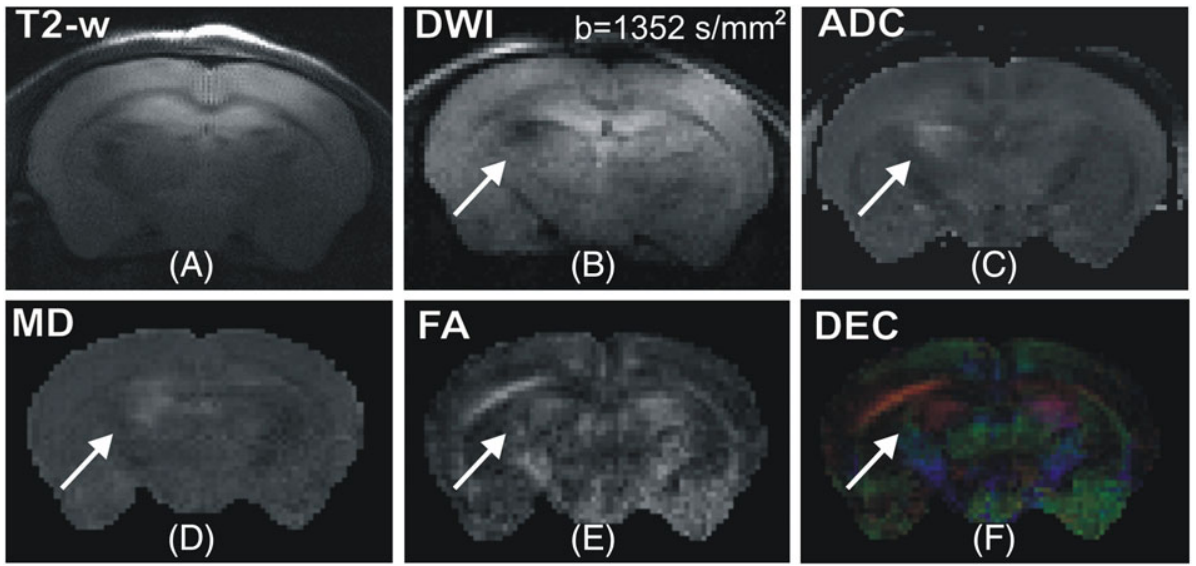

FIGURE 3 In vivo MRI of LN-2540GS glioma sphere xenograft, three months after injection of the cells. Upper panel: coronal $T_{2}$-weighted (A) and diffusion-weighted (B) images of the mouse brain with LN-2540GS xenograft NCH-1365, and the calculated ADC map (C). Lower panel: corresponding diffusion tensor maps, MD (D), FA (E) and FA-modulated DEC (F) maps. The lesion (arrow) is visible on the diffusion-weighted image (B), ADC (C) and DTI maps
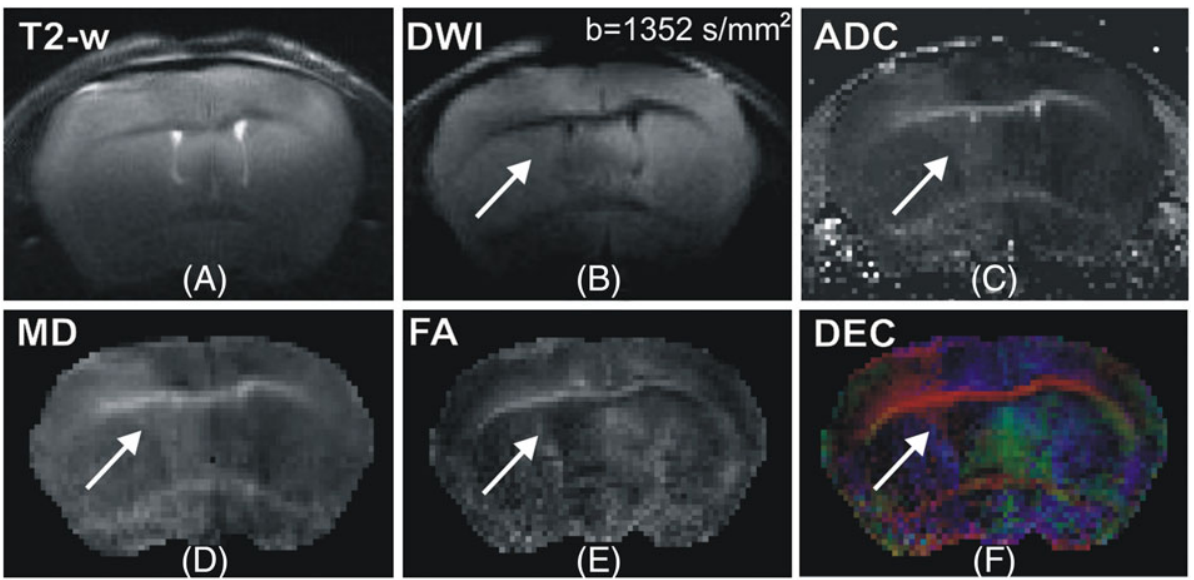

FIGURE 4 In vivo MRI of LN-2669GS glioma sphere xenograft, five months after injection of the cells. Upper panel: coronal $T_{2}$-weighted (A) and diffusion-weighted (B) images of the mouse brain with LN-2669GS xenograft NCH-1364, and the calculated ADC map (C). Lower panel: corresponding diffusion tensor maps, MD (D), FA (E) and FA-modulated DEC (F) maps. The lesion (arrow) is visible on the diffusion-weighted image (B) and ADC (C) and DTI maps. Due to the highly invasive and diffuse character of this tumour (corresponding histology, Figure $9 \mathrm{~A}$ ) it is difficult to delineate tumour borders
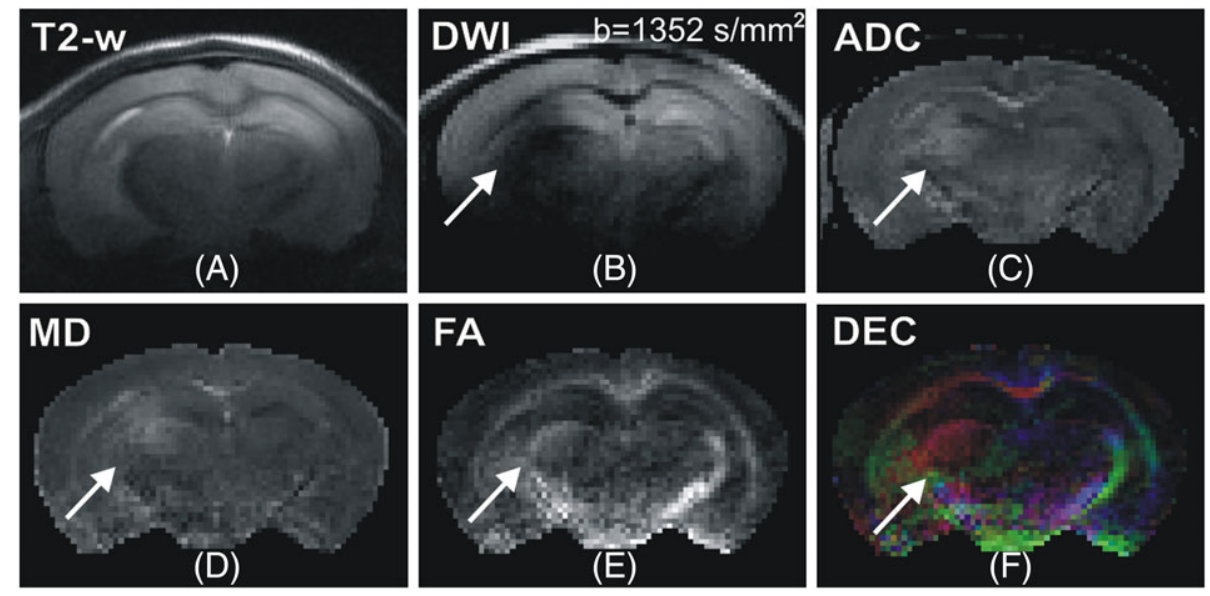

FIGURE 5 In vivo MRI of LN-2540GS glioma sphere xenograft, five months after injection of the cells. $T_{2}$-weighted (A) and diffusion-weighted (B) images, and the calculated ADC map (C); MD (D), FA (E) and FA-modulated DEC (F) maps of a coronal slice from the mouse brain with the LN-2540GS xenograft NCH-1365. MD, FA and DEC maps, computed after DTI reconstruction, show a lesion (indicated by the arrow) 


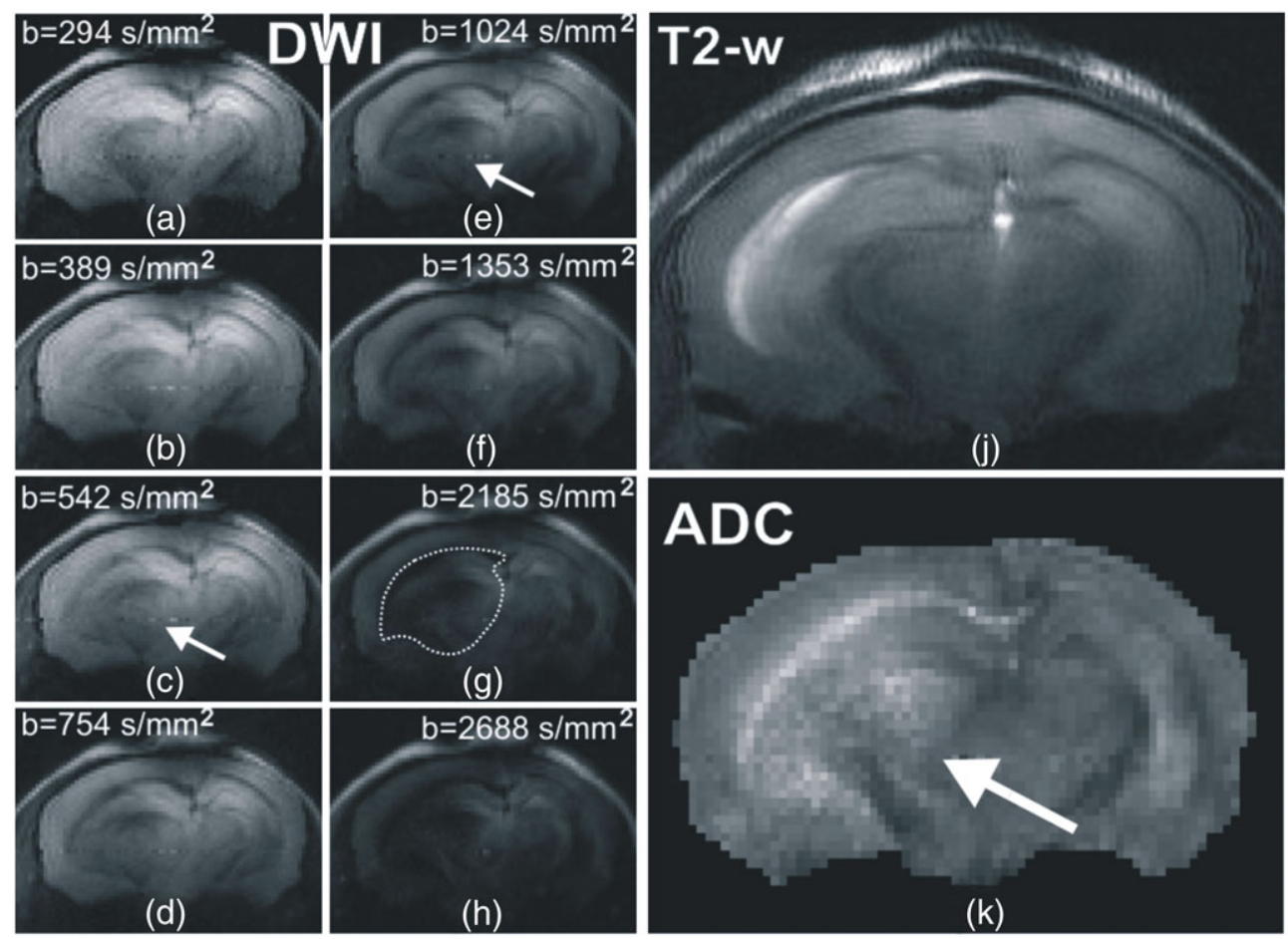

(A)

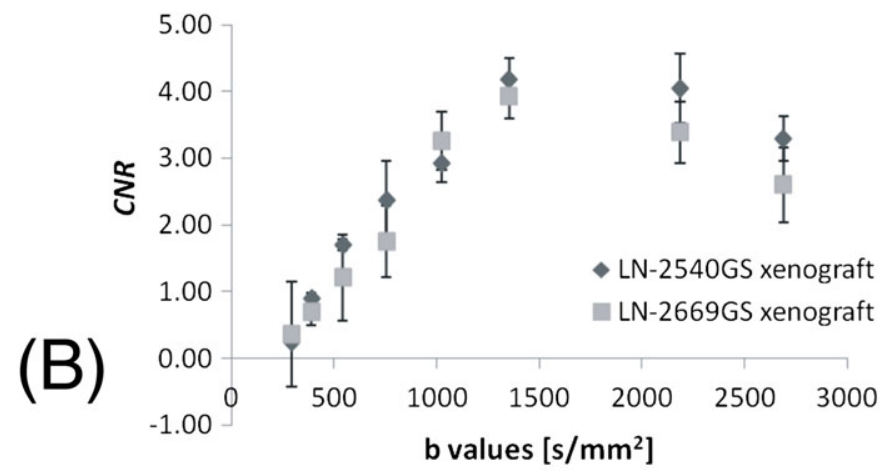

FIGURE 6 In vivo MRI of LN-2540GS glioma sphere xenograft (A) and CNR data of both xenografts (B), all acquired during the last MRI session (five months after injection of the cells). A, Left panel: coronal diffusion-weighted images (a-h) of the mouse brain with LN-2540GS xenograft $\mathrm{NCH}-1365$. Images are related to the DWI acquisition with $b$ values increasing along the readout direction. Right panel: corresponding anatomical $T_{2}$-weighted image (j) and ADC map ( $k$ ) of glioma-bearing xenograft. Images were acquired 15 days after those shown in Figure 5. A lesion (indicated by arrow and delineated by a dotted line in (g)) is visible on the diffusion-weighted images (c-h) and the ADC map. B, The CNR data of LN2669GS xenograft $\mathrm{NCH}-1364$ and LN-2540GS xenograft $\mathrm{NCH}-1365$ as a function of $b$ values obtained in the last MRI session

well as in the same brain regions of controls are summarized in Table 3 and Table 4 for both xenografts at early stages of tumour development (three months after injection) and at five months after cell injection, respectively.

For both stages of tumour growth, either three months or five months after cell injection (Table 3 and Table 4, respectively), a significant increase in MD was observed in tumours grown as LN-2669GS xenografts compared with those grown from LN-2540GS cells, with the $C L$ area and with the values obtained from the same brain regions of controls. On the other hand, a significant decrease in FA was observed in both cases (Table 3 and Table 4) in tumours grown as LN-2669GS xenograft, whereas no changes were observed in those grown from LN-2540GS cells compared with the CL area and the control. Moreover, ADC values increased similarly in tumours grown from each xenograft (LN-2669GS or LN-2540GS xenografts), either at three or five months after cell injection (Table 3 and Table 4, respectively), compared with those in the contralateral area and in the controls.

By comparing the corresponding values of the diffusion indices for each stage of tumour development, either three months (Table 3 ) or five months after cell injection (Table 4), the diffusion indices are slightly lower at earlier stages of tumour growth (Table 3). However, no significant difference was found between each corresponding diffusion indices in the two investigated stages of tumour development.

\section{2 | MRS results}

Proton spectra were measured immediately before sacrificing mice for histological assessment. In Figure $7,{ }^{1} \mathrm{H}$ spectra of a mouse brain from a 
TABLE 3 Summary of ADC values, FA and MD indices of tumour (T) and contralateral (CL) areas of both LN-2669GS and LN-2540GS xenografts at early stages of tumour development (3 months after cell injection). ADC values and DTI indices obtained from the corresponding brain regions of the control group are also reported. Data are mean \pm s.e.m.

\begin{tabular}{|c|c|c|c|c|c|}
\hline & \multicolumn{2}{|c|}{ LN-2669GS xenograft } & \multicolumn{2}{|c|}{ LN-2540GS xenograft } & \multirow[b]{2}{*}{ Control $^{\mathrm{a}}$} \\
\hline & $T$ & $\mathrm{CL}$ & $\mathrm{T}$ & $\mathrm{CL}$ & \\
\hline $\mathrm{ADC}\left[10^{-3} \mathrm{~mm}^{2} / \mathrm{s}\right]$ & $0.65 \pm 0.04^{b, c}$ & $0.53 \pm 0.06$ & $0.64 \pm 0.02^{b, c}$ & $0.54 \pm 0.05$ & $0.56 \pm 0.02$ \\
\hline $\mathrm{MD}\left[10^{-3} \mathrm{~mm}^{2} / \mathrm{s}\right]$ & $0.69 \pm 0.07^{b, c, d}$ & $0.58 \pm 0.05$ & $0.64 \pm 0.05^{\mathrm{b}, \mathrm{c}}$ & $0.58 \pm 0.03$ & $0.58 \pm 0.03$ \\
\hline
\end{tabular}

${ }^{a}$ Control data were computed from regions corresponding to tumour location, symmetrically in both hemispheres.

Statistics:

${ }^{\mathrm{b}}$ significantly different from $\mathrm{CL}$ area in the same group (paired two-tailed $t$ test, $p<0.01$ );

${ }^{\mathrm{c}}$ significantly different from the related brain regions in the controls (unpaired two-tailed $t$ test, $p<0.01$ ),

${ }^{d}$ significantly different from the corresponding value in tumour grown as LN-2540GS xenograft.

TABLE 4 Summary of ADC values, FA and MD indices of tumour (T) and contralateral (CL) area of both LN-2669GS and LN-2540GS xenografts at 5 months after cell injection. ADC values and DTI indices obtained from the corresponding brain regions of the control group are also reported.

Data are mean \pm s.e.m.

\begin{tabular}{|c|c|c|c|c|c|}
\hline & \multicolumn{2}{|c|}{ LN-2669GS xenograft } & \multicolumn{2}{|c|}{ LN-2540GS xenograft } & \multirow[b]{2}{*}{ Control $^{\mathrm{a}}$} \\
\hline & $\bar{T}$ & $\mathrm{CL}$ & $T$ & $\mathrm{CL}$ & \\
\hline $\mathrm{ADC}\left[10^{-3} \mathrm{~mm}^{2} / \mathrm{s}\right]$ & $0.67 \pm 0.07^{b, c}$ & $0.53 \pm 0.07$ & $0.65 \pm 0.04^{b, c}$ & $0.54 \pm 0.05$ & $0.54 \pm 0.02$ \\
\hline $\mathrm{MD}\left[10^{-3} \mathrm{~mm}^{2} / \mathrm{s}\right]$ & $0.71 \pm 0.04^{b, c, d}$ & $0.57 \pm 0.04$ & $0.66 \pm 0.05^{b, c}$ & $0.56 \pm 0.03$ & $0.57 \pm 0.03$ \\
\hline
\end{tabular}

${ }^{a}$ Control data were computed from regions corresponding to tumour location, symmetrically in both hemispheres.

Statistics:

${ }^{\mathrm{b}}$ significantly different from $\mathrm{CL}$ area in the same group (paired two-tailed $t$ test, $p<0.01$ );

${ }^{\mathrm{c}}$ significantly different from the related brain regions in the controls (unpaired two-tailed $t$ test, $p<0.01$ ),

${ }^{d}$ significantly different from the corresponding value in tumour grown as LN-2540GS xenograft.

control (Figure 7A), LN-2669GS xenograft NCH-1364 (Figure 7B) and LN-2540GS xenograft NCH-1365 (Figure 7C) are shown.

The spectra of tumours (Figure 7B,C) were acquired 5 months after injection of glioma sphere cells and compared with the control spectrum (Figure 7A). The main changes in metabolite concentrations (indicated by arrows) are depicted in Figure 7 and Figure 8.

In particular, the tumour grown from the LN-2669GS sphere line (Figure 7B) showed a decrease in N-acetylaspartate (NAA; $4.7 \mu \mathrm{mol} /$ g versus $7.7 \pm 0.4 \mu \mathrm{mol} / \mathrm{g}$ in controls, $p<0.01)$, glutamate (Glu; $4.7 \mu \mathrm{mol} / \mathrm{g}$ versus $8.4 \pm 0.4 \mu \mathrm{mol} / \mathrm{g}, p<0.001$ ), NAA + Nacetylaspartylglutamate (NAAG) $(5.7 \mu \mathrm{mol} / \mathrm{g}$ versus $8.6 \pm 0.4 \mu \mathrm{mol} / \mathrm{g}$, $p<0.01)$ and Glu + glutamine (Gln) $(8.2 \mu \mathrm{mol} / \mathrm{g}$ versus $10.8 \pm 0.4 \mu \mathrm{mol} / \mathrm{g}, p<0.05)$ concentrations, and an increase in myoinositol (Ins; $10.8 \mu \mathrm{mol} / \mathrm{g}$ versus $4.3 \pm 0.4 \mu \mathrm{mol} / \mathrm{g}, p<0.0001$ ) and glucose (Glc) $(4.7 \mu \mathrm{mol} / \mathrm{g}$ versus $1.5 \pm 0.3 \mu \mathrm{mol} / \mathrm{g}, p<0.01)$ ones (solid black bars, Figure 8).

The tumour grown from the LN-2540GS sphere line (Figure 7C) showed a more profound reduction in NAA $(1.8 \mu \mathrm{mol} / \mathrm{g}$ versus $7.7 \pm 0.4 \mu \mathrm{mol} / \mathrm{g}$ in controls, $p<0.0001)$, Glu $(3.7 \mu \mathrm{mol} / \mathrm{g}$ versus $8.4 \pm 0.4 \mu \mathrm{mol} / \mathrm{g}, p<0.0001), \mathrm{NAA}+\mathrm{NAAG}(2.6 \mu \mathrm{mol} / \mathrm{g}$ versus $8.6 \pm 0.4 \mu \mathrm{mol} / \mathrm{g}, p<0.0001)$ and $\mathrm{Glu}+\mathrm{Gln}(6.2 \mu \mathrm{mol} / \mathrm{g}$ versus $10.8 \pm 0.4 \mu \mathrm{mol} / \mathrm{g}, p<0.0001)$ concentrations and an increase in the Ins $(15.0 \mu \mathrm{mol} / \mathrm{g}$ versus $4.3 \pm 0.4 \mu \mathrm{mol} / \mathrm{g}, p<0.0001)$ and $\mathrm{Glc}$ (6.4 $\mu \mathrm{mol} / \mathrm{g}$ versus $1.5 \pm 0.3 \mu \mathrm{mol} / \mathrm{g}, p<0.0001)$, with additional changes in glycine (Gly; $5.0 \mu \mathrm{mol} / \mathrm{g}$ versus $1.2 \pm 0.1 \mu \mathrm{mol} / \mathrm{g}$, $p<0.001$ ) and taurine (Tau; $5.7 \mu \mathrm{mol} / \mathrm{g}$ versus $12.6 \pm 1.1 \mu \mathrm{mol} / \mathrm{g}$, $p<0.0001$ ), when compared with the LN-2669GS sphere line (solid grey bars, Figure 8).

\subsection{Immunohistochemical results}

In order to assess imaging and spectroscopic results, histological and immunohistochemical analysis was performed on each mouse. Due to the highly invasive nature of the human LN-2669GS cells, the intracerebral lesion was not visible on the H\&E section (Figure 9a). The hNCL immunostaining (Figure 9c,d) identifies the human tumour cells, and visualizes the infiltrative growth pattern of the human LN-2669GS tumour cells. GFAP expression indicated a region with enhanced gliosis that reached over to the co-lateral side, probably induced by tumour cell infiltration (Figure 9b). The area with strongest gliosis, as indicated by GFAP expression, shown in Figure 9b, correlates with the hypointense region in the diffusion-weighted image (Figure 4B), the hyperintense areas in the $A D C$ and $M D$ maps (Figure 4C,D) and the hypo-intense regions in the FA map (Figure 4E). Images shown in Figure 4 as well as the spectrum displayed in Figure 7B were all acquired during the last MRI session, immediately before euthanizing the mouse.

The LN-2540GS xenograft comprises a compact and a more invasive component visualized on the H\&E (Figure 9e) and the GFAP immunostained (Figure 9f) coronal section as well as by the nuclear expression of tumour protein 53 (TP53; not shown), which is detected in all tumour cells, in agreement with $\mathrm{hNCL}$ staining (Figure $9 \mathrm{~g}$ ). The high cell density in the compact tumour area is visualized in Figure 9h. 


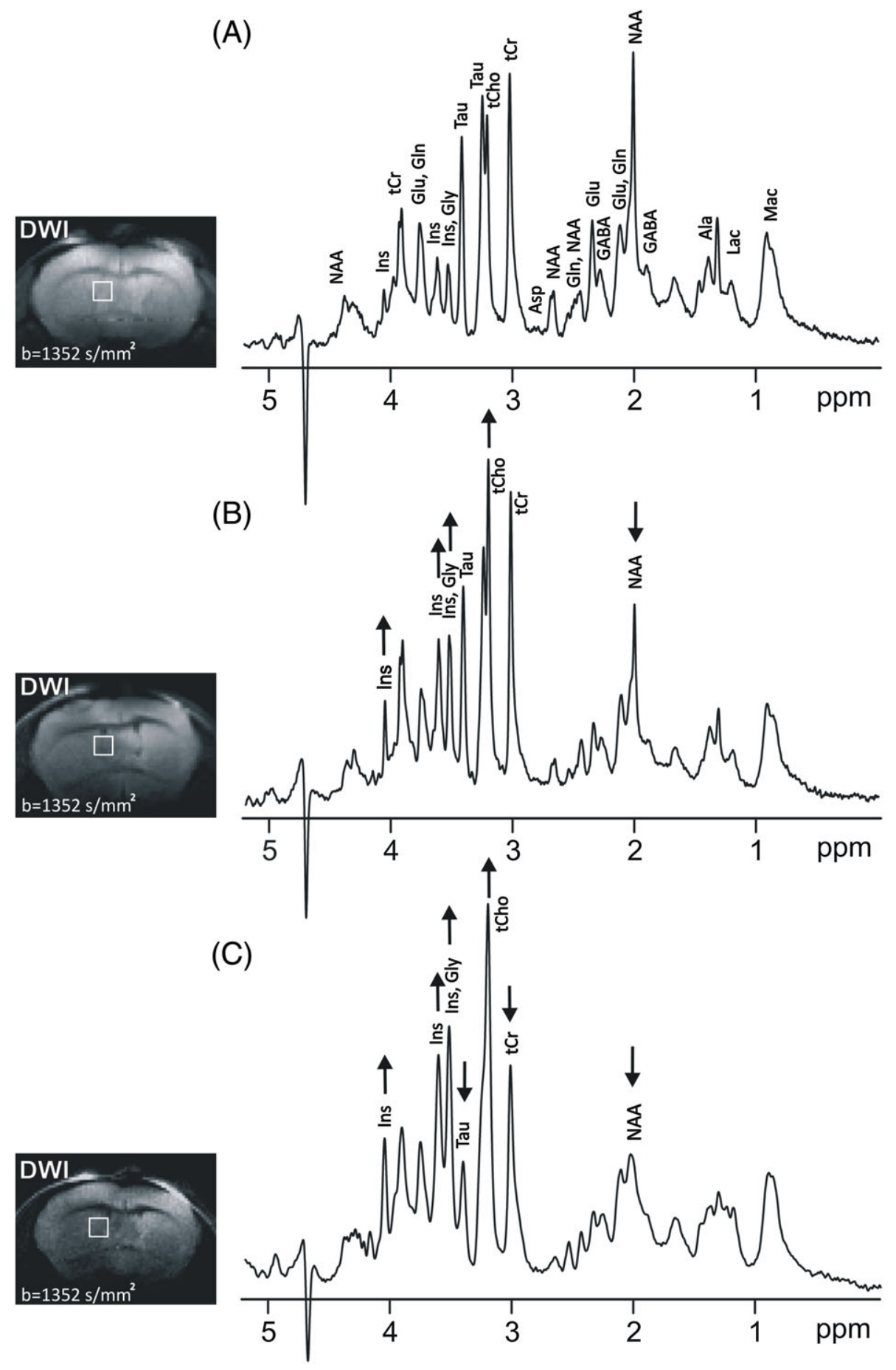

FIGURE $7{ }^{1} \mathrm{H}$ spectra of the brain from a control mouse (A) and from a tumour derived from LN-2669GS xenograft NCH-1364 (B), and a tumour derived from LN-2540GS xenograft NCH-1365 (C). Spectra B and C were acquired 5 months after cell injection. Spectra are displayed with a Gaussian filter (0.11)

The tumour area visible in Figure $9 \mathrm{e}-\mathrm{g}$ correlates with the hypointense regions visible on the DWI acquisition (Figure $6 \mathrm{c}-\mathrm{h}$ ) and the hyper-intense area shown in Figure 6k. Images shown in Figure 6A and the spectrum reported in Figure $7 C$ were all acquired during the last MRI session, immediately before the mice were euthanized.

It is of note that the two xenografts display a striking difference in cell density, as visualized in Figure 9d,h, displaying at high magnification an area of human tumour cells (with the same magnification): the LN-2669GS xenograft (Figure 9A, d) shows a lower tumour cell density than the LN-2540GS xenograft (Figure 9B, h).
In addition, none of the histological sections of both xenografts shows any areas of necrosis, in accordance with imaging results.

\section{4 | DISCUSSION}

We have developed a diffusion MR protocol at 14.1 T for early detection and investigation of human glioma xenografts in mice. Its performance was documented on human glioma xenografts derived from the sphere lines LN-2540GS and LN-2669GS, 


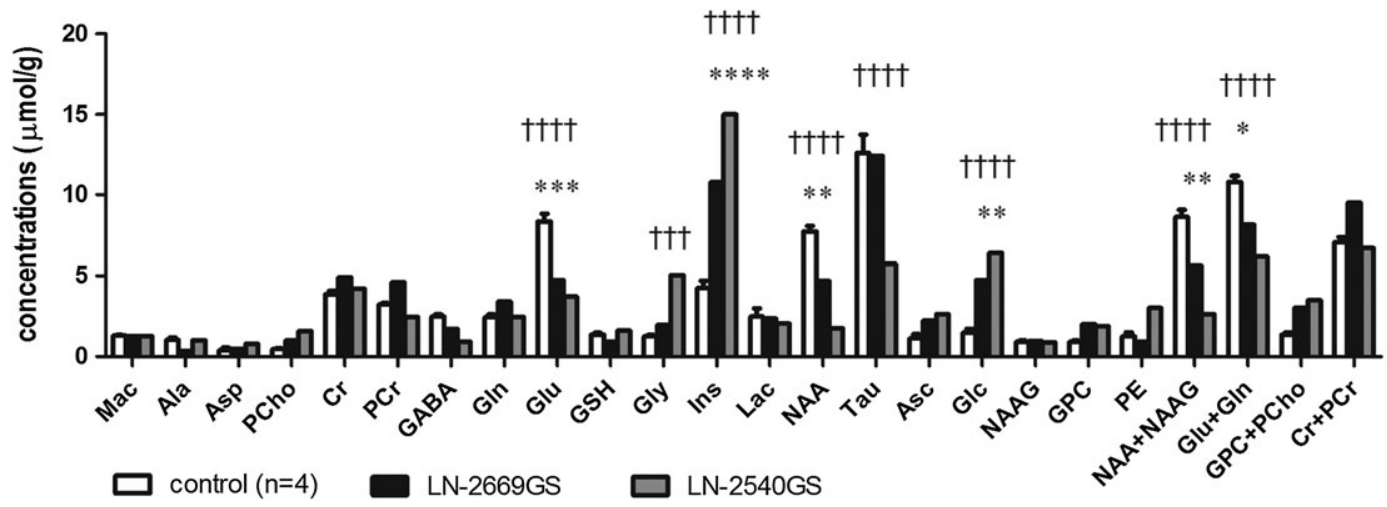

FIGURE 8 Absolute metabolite concentrations in the tumours grown from LN-2669GS and LN-2450GS sphere lines as well as in the same brain region of control mice obtained by in vivo MR spectroscopy 5 months after injection of the cells. Statistic: *Absolute metabolite concentrations from tumour grown from LN-2669GS NCH-1364 significantly different from those of the same brain region of controls $(p<0.05)$; ${ }^{* *} p<0.01$; ${ }^{* * *} p<0.001 ;{ }^{* * *} p<0.0001 ;{ }^{\dagger \dagger}$ absolute metabolite concentrations from tumour grown from LN-2540GS NCH-1365 significantly different from those of the same brain region of controls $(p<0.001)$; ${ }^{\text {tt† }} p<0.0001$

$H \& E$

(A)

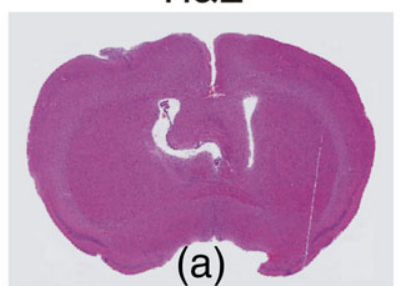

(B)

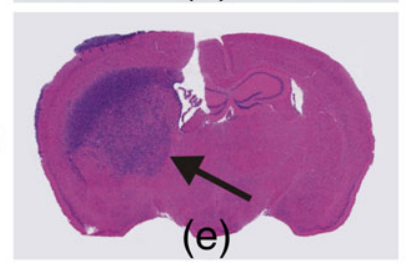

GFAP

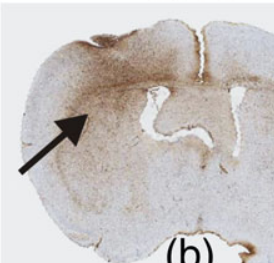

(b) ?

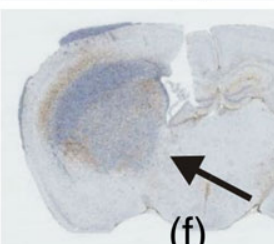

$\mathrm{hNCL}$

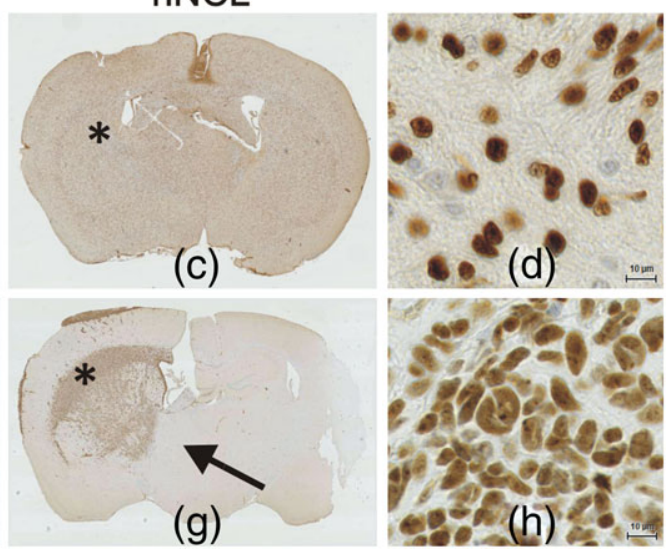

FIGURE 9 Histology of the LN-2669GS and LN-2540GS derived xenografts. For both xenografts full brain sections are shown for H\&E stain (a,e), and immunohistochemistry for GFAP (b,f), and the human specific NCL protein (c,d,g,h). A, In the LN-2669GS xenograft (NCH-1364), the H\&E stained section (a) does not reveal the lesion, with the exception of a small compact tumour part at the surface of the brain due to reflux at the injection site. The GFAP (b) expression (arrow) indicates gliosis induced by infiltrating tumour cells. The visualization of the human tumour cells by $\mathrm{hNCL}(\mathrm{c}, \mathrm{d})$ indicates the highly invasive growth properties of the LN-2669GS xenograft NCH-1364 (magnification of the marked area ${ }^{*}$ ), scale bar 10 $\mu \mathrm{m})$. B, The LN-2540GS xenograft is readily visible with $\mathrm{H} \& \mathrm{E}$, identifying a compact and an invasive part (e). Magnification of the marked area $\left({ }^{*}\right)$ in the hNCL immunostained section (h) indicates high cell density in the most compact part of the LN-2540GS xenograft NCH-1365 (scale bar $10 \mu \mathrm{m}$ )

characterized by their slow and diffuse infiltrative growth in the mouse brain. ${ }^{8}$ With the optimized acquisition parameters of the diffusion-weighted stimulated-echo (PGSTE) sequence, in particular the diffusion time $(\Delta=80 \mathrm{~ms}$ ), the protocol allowed a proper detection and investigation of both xenograft models in the regime of hindered diffusion.

Different diffusion times ( $\Delta=40-220 \mathrm{~ms}$ ) were explored in a pilot study carried out on LN-2540GS glioma sphere xenografts at early stages of growth, and the best tumour contrast was found for $\Delta=80 \mathrm{~ms}$ (Table 2). By selecting this observation time, the diffusion distance $(I \sim 10 \mu \mathrm{m})$ was comparable to the average distance between glioma sphere cells, which were not densely packed within the investigated xenografts due to their diffuse infiltrative growth. Therefore, shorter $\Delta$ does not give a proper tumour contrast, whereas $\Delta=80 \mathrm{~ms}$, by probing the distance between glioma sphere cells, optimizes tumour contrast for the investigated xenografts.
Different diffusion properties due to diverse tumour microstructure in each model were also demonstrated by the calculated diffusion indices. Finally, the presence of tumours was confirmed by localized spectroscopy and by immunohistochemical analysis, which also provided information on morphologic tumour properties.

As it is well known ${ }^{38}$ the advantage of the PGSTE sequence is the use of long diffusion times, avoiding signal loss due to the transverse relaxation time $T_{2}$. On the other hand, its main disadvantage is a loss of $50 \%$ of the MR signal. ${ }^{42}$ We partly compensated this loss by using the shortest possible $T_{\mathrm{E}}(22 \mathrm{~ms})$, an ultra-high magnetic field and a small surface coil (dimensions of $21 \mathrm{~mm} \times 14 \mathrm{~mm}$ ). However, the increase in SNR due to the high magnetic field was reduced by shorter transverse relaxation time $T_{2}$. In addition, larger susceptibility effects due to the high magnetic field made the detection of glioma sphere xenografts even more challenging.

Tumours grown in both xenograft models were not visible on $T_{2}$-weighted images (Figures 2A, 3A, 4A, 5A and 6j), even though 
a ventricular enlargement indicating tumour presence was observed on the $T_{2}$-weighted image (Figure 6 ) of the mouse brain with the LN-2540GS xenograft. Detection of slow-growing tumours from glioma-initiating cells was also difficult using contrast-enhanced $\mathrm{T}_{1}$-weighted $\mathrm{MRI}^{34}$

During investigation of glioma sphere xenografts by means of diffusion MRI, the first evidence of tumour presence on diffusionweighted images was revealed three months after glioma sphere cell injection for both xenograft models (Figure 2 and Figure 3). The absence of signs of necrosis and haemorrhagic foci in MR images, which was confirmed by histology, underlined the problematic detection of these slowly growing tumours using standard MRI techniques.

Different diffusion properties between glioma xenografts grown from LN-2669GS and LN-2540GS, respectively, were observed at three months (Table 3 ) and five months after cell injection (Table 4). At both time points, the larger MD in the LN-2669GS tumour model indicates that water molecules diffuse faster in tumours grown as LN-2669GS xenografts than in those grown from LN-2540GS cells that, in addition to the infiltrative part, comprise a compact tumour component. Faster diffusion behaviour in the tumour grown from LN-2669GS compared with LN-2540GS is probably explained by the much lower cell density of the former (Figure 9d,h). Moreover, the reduced FA in the LN-2669GS model suggests loss of anisotropy in this tumour compared with the one grown from LN-2540GS cells, indicating a looser and less anisotropic tumour microstructure in the LN-2669GS engraft, which is also in accordance with histology. However, the six gradient orientations used in this study, as described in the method section, do not provide an optimal sampling scheme for obtaining detailed information from the FA maps.

The growth properties of the glioma sphere cell line-derived xenografts in this study were highly invasive, without formation of central necrosis. These properties allowed the investigation of invasive tumour characteristics, which are a major diagnostic problem in patients with GBM and low grade gliomas, to determine the extent of the tumour requiring treatment. Thus, our imaging results suggest that diffusion techniques can be more successful than standard $T_{2}$-weighted $M R$ images in detecting infiltrative tumours growing slowly as xenografts in mouse brain.

The comparison between ${ }^{1} \mathrm{H}$ spectra of the two investigated xenograft models shows more pronounced changes in absolute metabolite concentrations in tumours derived from LN-2540GS than in those from the LN-2669GS sphere line (Figure 7 and Figure 8). This may be explained by different characteristics of these tumours. Since spectra of both xenografts were measured at the same time point after cell injection (5 months), the spectral profile of tumours grown from LN-2540GS sphere line may indicate faster growing tumours compared with those grown as LN-2669GS xenograft. The diffusion results support this finding, as shown by comparing MR images between the two xenografts at three months (Figure 2 and Figure 3, respectively) and five months after cell injection (Figure 4 and Figure 5). Moreover, the faster growing tumours, LN-2540GS xenografts, are characterized by a lower MD compared with that of LN-2669GS xenografts. This is consistent with a more compact

tumour for the LN-2540GS xenograft compared with that grown from LN-2669GS sphere lines, which is in accordance with tumour histology.

\section{5 | CONCLUSION}

In vivo measurements performed in this study showed that the diffusion MRI technique is a useful method to detect and follow slowly growing, diffuse infiltrative tumours in the mouse brain. Imaging data in combination with localized proton spectra of lesions and immunohistochemical assessment demonstrated that the optimized diffusion MRI protocol can be useful in early detection of slow-growing human glioma xenografts as well as in the investigation of diffusion properties of tumours during their growth. Moreover, the calculated diffusion indices in the tumours reflected microstructural differences between LN-2669GS and LN-2540GS xenografts, confirmed by histology. The diffusion parameters measured at a moderately long diffusion time (i.e. $\Delta=80 \mathrm{~ms}$ ) can be considered as potential biomarkers for early detection of diffuse infiltrative gliomas in humans due to the possible implementation of the proposed protocol on the clinical MR scanners.

\section{ACKNOWLEDGEMENTS}

We gratefully acknowledge Professor Andrew M. Blamire for his helpful comments and suggestions.

We thank Dr Nicholas Kunz for his valuable advice and help during data acquisition.

This study was supported by Centre d'Imagerie BioMédicale (CIBM) of the UNIL, UNIGE, HUG, CHUV, EPFL and the Leenaards and Jeantet Foundations, and the Swiss National Science Foundation (31003 A-138116 to MEH).

\section{ABBREVIATIONS USED}

$\delta$

$\Delta$

ADC

CNR

DEC

DTI

DWI

FA

GBM

GFAP

Glc

Gln

Glu

Gly

H\&E

Ins

$I=(2 \mathrm{D} \Delta)^{1 / 2}$

$M A B$

$M D$

NAA gradient-pulse duration

gradient-pulses separation, diffusion time

apparent diffusion coefficient

contrast-to-noise ratio

directionally encoded colour

diffusion tensor imaging

diffusion-weighted imaging

fractional anisotropy

glioblastoma multiforme

glial fibrillary acidic protein

glucose

glutamine

glutamate

glycine

haematoxylin and eosin

myo-inositol

diffusion distance

monoclonal antibody

mean diffusivity

$\mathrm{N}$-acetylaspartate 


$\begin{array}{ll}\text { NAAG } & \text { N-acetylaspartylglutamate } \\ \text { NCL } & \text { nucleolin } \\ \text { PGSTE } & \text { pulse gradient stimulated echo } \\ \text { ROI } & \text { region of interest } \\ \text { SNR } & \text { signal-to-noise ratio } \\ \text { Tau } & \text { taurine } \\ \text { tCho } & \text { total choline } \\ \text { tCr } & \text { total creatine } \\ \text { VOI } & \text { volume of interest. }\end{array}$

\section{REFERENCES}

1. Claes A, Idema AJ, Wesseling P. Diffuse glioma growth: a guerrilla war. Acta Neuropathol. 2007;114(5):443-458.

2. Wen PY, Kesari S. Malignant gliomas in adults. N Engl J Med. 2008; 359(5):492-507.

3. Louis DN, Perry A, Reifenberger G, et al. The 2016 World Health Organization classification of tumours of the central nervous system: a summary. Acta Neuropathol. 2016;131(6):803-820.

4. Stupp R, Mason WP, van den Bent MJ, et al. European Organisation for Research and Treatment of Cancer Brain Tumor and Radiotherapy Groups; National Cancer Institute of Canada Clinical Trials Group. Radiotherapy plus concomitant and adjuvant temozolomide for glioblastoma. N Engl J Med. 2005;352(10):987-996.

5. Ceccarelli M, Barthel FP, Malta MT, et al. Molecular profiling reveals biologically discrete subsets and pathways of progression in diffuse glioma. Cell. 2016;164(3):550-563.

6. Barth RF, Kaur B. Rat brain tumour models in experimental neurooncology: the C6, 9 L, T9, RG2, F98, BT4C, RT-2 and CSN-1 gliomas. J Neurooncol. 2009;94(3):299-312.

7. Huszthy PC, Daphu I, Niclou SP, et al. In vivo models of primary brain tumors: pitfalls and perspectives. Neuro Oncol. 2012;14(8):979-993.

8. Vassallo I, Zinn P, Lai M, Rajakannu P, Hamou MF, Hegi ME. WIF1 reexpression in glioblastoma inhibits migration through attenuation of non-canonical WNT signaling by downregulating the IncRNA MALAT1. Oncogene. 2016;35(1):12-21.

9. Huse JT, Holland EC. Genetically engineered mouse models of brain cancer and the promise of preclinical testing. Brain Pathol. 2009; 19(1):132-143.

10. Dhermain FG, Hau P, Lanfermann $\mathrm{H}$, Jacobs $\mathrm{AH}$, van den Bent $\mathrm{MJ}$. Advanced MRI and PET imaging for assessment of treatment response in patient with gliomas. Lancet Neurol. 2010;9:906-920.

11. Huang RY, Neagu MR, Reardon DA, Wen PY. Pitfalls in the neuroimaging of glioblastoma in the era of antiangiogenic and immuno/targeted therapy - detecting illusive disease, defining response. Front Neurol. 2015;6:33

12. Porcari $\mathrm{P}$, Capuani S, D'Amore $\mathrm{E}$, et al. In vivo ${ }^{19} \mathrm{~F} \mathrm{MRI}$ and ${ }^{19} \mathrm{~F}$ MRS of ${ }^{19} \mathrm{~F}$-labelled boronophenylalanine-fructose complex on $\mathrm{C} 6$ rat glioma models to optimize boron neutron capture therapy (BNCT). Phys Med Biol. 2008;53(23):6979-6989.

13. Padhani AR, Liu G, Koh DM, et al. Diffusion-weighted magnetic resonance imaging as a cancer biomarker: consensus and recommendations. Neoplasia. 2009;11(2):102-125.

14. Peet AC, Arvanitis TN, Leach MO, Waldman AD. Functional imaging in adult and paediatric brain tumours. Nat Rev Clin Oncol. 2012; 9(12):700-711.

15. Price SG, Jillard JH. Imaging biomarkers of brain tumour margin and tumour invasion. Br J Radiol. 2011; Spec No2:S159-S167.

16. Durst CR, Raghavan P, Shaffrey ME, et al. Multimodal MR imaging model to predict tumor infiltration in patients with gliomas. Neuroradiology. 2014;56(2):107-115.
17. On N, Mitchell R, Savant SD, Bachmeier CJ, Hatch GM, Miller DW. Examination of blood-brain barrier (BBB) integrity in a mouse tumour model. J Neurooncol. 2013;111(2):133-143.

18. Ganslandt O, Stadlbauer A, Fahlbusch R, et al. Proton magnetic resonance spectroscopic imaging integrated into image-guided surgery: correlation to standard magnetic resonance imaging and tumor cell density. Neurosurgery. 2005;56(Suppl 2):291-298.

19. Kalpathy-Cramer I, Gerstner ER, Emblem KE, Andronesi OC, Rosen B. Advanced magnetic resonance imaging of the physical process in human glioblastoma. Cancer Res. 2014;74(17):4622-4637.

20. Jones DK (ed.). Diffusion MRI: Theory, Methods, and Applications, vol 1. Oxford: Oxford University Press; 2010.

21. Wang S, Zhou J. Diffusion tensor magnetic resonance imaging of rat glioma models: a correlation study of MR imaging and histology. $J$ Comput Assist Tomogr. 2012;36(6):739-744.

22. Hoff BA, Chenevert TL, Bhojani MS, et al. Assessment of multiexponential diffusion features as MRI cancer therapy response metrics. Magn Reson Med. 2010;64(5):1499-1509.

23. Yablonskiy DA, Sukstanskii AL. Theoretical models of the diffusion weighted MR signal. NMR Biomed. 2010;23(7):661-681.

24. Fieremans E, Novikov DS, Jensen JH, Helpern JA. Monte Carlo study of a two-compartment exchange model of diffusion. NMR Biomed. 2010;23(7):711-724.

25. Pfeuffer J, Flogel U, Dreher W, Leibfritz D. Restricted diffusion and exchange of intracellular water: theoretical modelling and diffusion time dependence of ${ }^{1} \mathrm{H}$ NMR measurements on perfused glial cells. NMR Biomed. 1998;11(1):19-31.

26. Basser PJ. Inferring microstructural features and the physiological state of tissues from diffusion-weighted images. NMR Biomed. 1995; 8(7/8):333-344.

27. Basser PJ, Mattiello J, Le Bihan D. MR diffusion tensor spectroscopy and imaging. Biophys J. 1994;66:259-267.

28. Basser PJ, Pierpaoli C. Microstructural and physiological features of tissues elucidated by quantitative-diffusion-tensor MRI. J Magn Reson. 1996;213(2):560-570.

29. Bennet KM, Hyde JS, Rand SD, et al. Intravoxel distribution of DWI decay rates reveals C6 glioma invasion in rat brain. Magn Reson Med. 2004;52(5):994-1004.

30. Lope-Piedrafita S, Garcia-Martin ML, Galons JP, Gillies RJ, Trouard TP. Longitudinal diffusion tensor imaging in a rat brain glioma model. NMR Biomed. 2008;21(8):799-808.

31. Maier SE, Sun Y, Mulkern RV. Diffusion imaging of brain tumors. NMR Biomed. 2010;23(7):849-864.

32. Kallenberg K, Goldmann T, Menke J, et al. Glioma infiltration of corpus callosum: early signs detected by DTI. J Neurooncol. 2013;112(2):217222.

33. Painter KJ, Hillen T. Mathematical modelling of glioma growth: the use of diffusion tensor imaging (DTI) data to predict the anisotropic pathways of cancer invasion. J Theor Biol. 2013;323:25-39.

34. Mlynárik V, Cudalbu C, Clément V, Marino D, Radovanovic I, Gruetter R. In vivo metabolic profiling of glioma-initiating cells using proton magnetic resonance spectroscopy at 14.1 Tesla. NMR Biomed. 2012; 25(4):506-513.

35. Bady P, Diserens AC, Castlella V, et al. DNA fingerprinting of glioma cell lines and considerations on similarity measurements. Neuro Oncol. 2012;14(6):701-711.

36. Sciuscio D, Diserenc AC, van Dommelem K, et al. Extent and patterns of MGMT promoter methylation in glioblastoma- and respective glioblastoma-derived spheres. Clin Cancer Res. 2011;17(2):255-266.

37. Gruetter R, Tkáč I. Field mapping without reference scan using asymmetric echo-planar techniques. Magn Reson Med. 2000;43(2):319-323.

38. Tanner JE. Use of stimulated-echo in NMR diffusion studies. J Chem Phys. 1970;52:2523-2526. 
39. Mlynárik V, Gambarota G, Frenkel H, Gruetter R. Localized shortecho-time proton MR spectroscopy with full signal-intensity acquisition. Magn Reson Med. 2006;54(5):965-970.

40. Tkáč I, Starčuk Z, Choi IY, Gruetter R. In vivo ${ }^{1} \mathrm{H}$ NMR spectroscopy of rat brain at $1 \mathrm{~ms}$ echo time. Magn Reson Med. 1999; 41(4):649-656.

41. Provencher SW. Estimation of metabolite concentrations from localized in vivo proton NMR spectra. Magn Reson Med. 1993; 30(6):672-679.

42. Nair G, Tanahashi Y, Low HP, Billings-Gagliardi S, Schwartz WJ, Duong TQ. Myelination and long diffusion times alter diffusion-tensor-imaging contrast in myelin-deficient shiverer mice. Neuroimage. 2005;28 (1):165-174.

How to cite this article: Porcari, P., Hegi, M. E., Lei, H., Hamou, M-F., Vassallo, I., Capuani, S., Gruetter, R., and Mlynarik, V. (2016), Early detection of human glioma sphere xenografts in mouse brain using diffusion MRI at 14.1 T, NMR Biomed., doi: 10.1002/nbm.3610 